\title{
ON A CONJECTURE OF DEMAILLY AND KOLLÁR*
}

\author{
D. H. PHONG ${ }^{\dagger}$ AND JACOB STURM ${ }^{\ddagger}$
}

1. Introduction. If $f$ is holomorphic in a neighborhood of a compact set $K$ in a complex manifold, define $c_{K}(f)$ to be the supremum of all real numbers $c$ such that $|f|^{-2 c}$ is integrable on some neighborhood of $K$.

In their recent paper [DK], Demailly and Kollár made the following remarkable conjectures.

Conjecture A: Fix the compact set $K$. For every non-zero holomorphic function $f$ and for every compact set $L$ containing $K$ in its interior, there is a number $\alpha=$ $\alpha(f, K, L)>0$ such that

$$
\sup _{L}|g-f|<\alpha \quad \Longrightarrow \quad c_{K}(g) \geq c_{K}(f)
$$

Conjecture B: Let

$$
\mathcal{C}(n)=\left\{c_{0}(f): f \text { is holomorphic in a neighborhood of the origin of } \mathbf{C}^{n}\right\}
$$

Then $\mathcal{C}(n)$ satisfies the ascending chain condition: every convergent increasing sequence in $\mathcal{C}(n)$ is stationary.

A version of Conjecture B in algebraic geometry has been formulated earlier, starting with the 1992 work of Shokurov [Sh][K1-2]. This algebraic geometric version of Conjecture B has been established in dimension $n=2$ by Shokurov in [Sh], and in dimension $n=3$ by Alexeev in [A]. Related conjectures and results in algebraic geometry can be found in [K1-2]. The exponents $c_{0}(f)$ also play an important role in the study of the existence of Kähler-Einstein metrics [CY][TY1-2][Si][T1-2] [Y12][DK].

In [DK], Demailly and Kollár had proved the following weaker version of Conjecture $A$ : under the same conditions, for any $\epsilon>0$, there exists a number $\alpha(f, K, L, \epsilon)$ such that

$$
\sup _{L}|g-f|<\alpha(f, K, L, \epsilon) \quad \Longrightarrow \quad c_{K}(g) \geq c_{K}(f)-\epsilon
$$

In dimension $n=2$, this last statement had also been obtained in [T2] and in [PS]. The purpose of this short note is to show that the methods of [PS] can also give the following theorem:

Theorem. Conjectures $A$ and $B$ hold when $n=2$.

It was already observed in $[\mathrm{DK}]$ that Conjecture $A$ follows from Conjecture $B$ combined with the weaker statement $\left(A_{\epsilon}\right)$. We shall nevertheless give direct separate proofs for both conjectures, since in our approach, the method of proof of Conjecture $A$ is no different from that of $\left(A_{\epsilon}\right)$. It will also emerge from our proof that $x$ is a limit

* Received May 11, 2000; accepted for publication July 4, 2000.

† Department of Mathematics, Columbia University, New York, NY 10027, USA (phong@math. columbia.edu).

$\ddagger$ Department of Mathematics, Rutgers University, Newark, NJ 08903, USA (sturm@andromeda. rutgers.edu). 
point of $\mathcal{C}(2)$ if and only if $x=0$ or $x$ is a number of the form $2 / a$ where $a$ is a positive integer.

2. Proof of Conjecture $\mathbf{A}$ when $n=2$. In [PS], an approach was developed for the study of integrals of $|f|^{-2 c}$ in $n$ variables, by iterating sharp estimates for one-dimensional integrals of the form

$$
\int_{B_{\Lambda}} \frac{\sum_{i=1}^{I}\left|P_{i}(z)\right|^{\epsilon}}{\sum_{j=1}^{J}\left|Q_{j}(z)\right|^{\delta}} d V
$$

Here $P_{i}(z)$ and $Q_{j}(z)$ are polynomials in the variable $z \in \mathbf{C}, \epsilon$ and $\delta$ are nonnegative real numbers, $d V=d x d y$ is the Euclidian measure on $\mathbf{C}$, and $B_{\Lambda}$ is the open disk of radius $\Lambda$. A key result was that the finite-dimensional space of polynomials $P_{i}(z), Q_{j}(z)$ admits a stratification into algebraic varieties, on each of which the size of the above integral is given by expressions of the form

$$
\frac{\left(\sum_{i=1}^{\hat{I}}\left|\hat{P}_{i}\left(B_{1}, \cdots, B_{M}\right)\right|^{2}\right)^{\hat{\epsilon}}}{\left(\sum_{j=1}^{\hat{J}}\left|\hat{Q}_{j}\left(B_{1}, \cdots, B_{M}\right)\right|^{2}\right)^{\hat{\delta}}}
$$

where $\hat{P}_{i}\left(B_{1}, \cdots, B_{M}\right)$ and $\hat{Q}_{j}\left(B_{1}, \cdots, B_{M}\right)$ are polynomials in the coefficients $B_{1}$, $\cdots, B_{M}$ of the original polynomials $P_{i}(z)$ and $Q_{j}(z)$ [PS, Theorem 4].

In this note we require the special case of these formulas, when the integrand in (2.0) reduces to $|Q(z)|^{-\delta}$ with $Q(z)$ a polynomial of degree $N$, and $\delta$ is a real number in the range $2 / N<\delta<4 / N$ such that $2 / \delta$ in non-integral. In this case the formulas simplify substantially, and we have [PS, Theorems 2 and 3]

Proposition 1. Fix a positive integer $N$. For every $r$ in the range $1 \leq r \leq$ $(N / 2)$, there exist polynomials $D_{r, j} \in \mathbf{Z}\left[A_{1}, \ldots, A_{N}\right]$, with $1 \leq j \leq h(r)=N ! /(2 r)$ ! with the following property. If we let

$$
\Delta_{r}=\Delta_{r}\left(a_{1}, \ldots, a_{N}\right)=\sum_{q=1}^{h(r)}\left|D_{r, q}\left(a_{1}, \ldots, a_{N}\right)\right|^{1 / h(r) !}
$$

then for all real numbers $\delta \in(2 /(N-r+1), 2 /(N-r))$ and every positive real number $\Lambda$ we have

$$
\int_{B_{\Lambda}} \frac{1}{|Q(z)|^{\delta}} d V \sim \frac{1}{\Delta_{r+1}^{(N-r+1) \delta-2} \Delta_{r}^{2-(N-r) \delta}}
$$

for all monic polynomials $Q(z)=\sum_{i=0}^{N} a_{i} z^{N-i}$ whose roots lie in $B_{\Lambda / 2}$. If $\delta<2 / N$ then

$$
\int_{B_{\Lambda}} \frac{1}{|Q(z)|^{\delta}} d V \sim \Lambda^{2-N \delta}
$$

The implied constants in (2.1) and (2.2) depend on $\delta$ and $\Lambda$, but they are independent of the coefficients of $Q$.

We can establish now Conjecture $A$ in 2 dimensions. It suffices to establish it when $K$ is a single point 0 . Let $f(z, w)$ be a holomorphic function in a neighborhood of the origin in $\mathbf{C}$ and assume $f(0)=0, f(z, w)$ is not identically zero. Let $M$ be the order of vanishing of $f(z, w)$ at the origin, i.e., the lowest degree with a non-vanishing monomial in the Taylor expansion of $f(z, w)$ at 0 . Then, after a suitable rotation of 
coordinates, the Weierstrass Preparation Theorem says that on some polydisk $U \times V$ centered at the origin, $f$ can be factored as $f=u_{f} p_{f}$ where $u_{f}$ is nowhere vanishing holomorphic function and

$$
p_{f}(z, w)=w^{M}+a_{1}(z) w^{M-1}+\cdots a_{M}(z) .
$$

Here $a_{i}(z)=a_{i, f}(z)$ are holomorphic functions satisfying the condition

$$
a_{i}(0)=0 .
$$

Moreover, there is a polydisk $U^{\prime} \times V^{\prime} \subseteq U \times V$, centered at the origin, and an $\alpha>0$ such that if $g$ is holomorphic on $U \times \bar{V}$ and if $\sup _{U \times V}|g-f|<\alpha$, then $g$ can be factored as $g=u_{g} p_{g}$ on $U^{\prime} \times V^{\prime}$ where $u_{g}$ is a holomorphic function such that $\left|u_{g}\right|$ is bounded below by a positive constant, and

$$
p_{g}(z, w)=w^{M}+a_{1, g}(z) w^{M-1}+\cdots a_{M, g}(z)
$$

Here the $a_{i, g}$ are holomorphic on $V^{\prime}$, although they may no longer satisfy (2.3). The map $g \rightarrow a_{i, g}$ (resp. $g \rightarrow u_{g}$ ) is continuous with respect to the sup norm metrics on $U \times V$ and $V^{\prime}$ (resp. $U \times V$ and $U^{\prime} \times V^{\prime}$ ). Thus we can choose $\alpha$ and $U^{\prime}$ sufficiently small so that $\|g-f\|=\sup _{U \times V}|g-f|<\alpha$ implies that $u_{g} u_{f}^{-1} \sim 1$ and for every $z \in U^{\prime}$, all the roots of $p_{g}(z, w)=0$ are in $\frac{1}{2} V^{\prime \prime}$ (see e.g. Lemma 5.2 in [PS]).

Let $N=2 M$ and define $b_{i, g}(z)$ by the formula

$$
p_{g}(z, w)^{2}=\sum_{i=0}^{N} b_{i, g}(z) w^{N-i}
$$

For every $r$ in the range $1 \leq r \leq M$, and for every holomorphic function $g$ satisfying $\|g-f\|<\alpha$, let $F_{r, g}(z)=\bar{\Delta}_{r}\left(b_{1, g}, \ldots, b_{N, g}\right)$, and let $n(r, g)$ be the unique real number such that

$$
|z|^{-n(r, g)} F_{r, g}(z)
$$

is continuous and non-vanishing at the origin. Proposition 1 implies that

$$
n(r, g)(N !) ! \in \mathbf{Z} .
$$

By shrinking $\alpha$ and $U^{\prime}$ even further, we may assume (using, for example, the winding number principle) that for all $g$ satisfying $\|g-f\|<\alpha$, and for all $r$, we have $n(r, g) \leq$ $n(r, f)$.

Let

$$
\Sigma(f)=\left\{c \in \mathbf{R}:|f|^{-2 c} \text { is integrable on some neighborhood of the origin }\right\} .
$$

It is well-known that $\Sigma(f)$ is an open set (using for example Hironaka's theorem on resolution of singularities), and thus $\Sigma(f)=\left(-\infty, c_{0}(f)\right)$. We must prove that if $\|g-f\|<\alpha$, then $\delta \in \Sigma(f)$ implies $\delta<c_{0}(g)$. In fact, if $T$ is at most a countably infinite set of real numbers, it suffices to prove that $\delta \in \Sigma(f) \backslash T$ implies $\delta<c_{0}(g)$.

Lemma 5.1 of [PS] guarantees that $\Sigma(f) \subseteq(0,4 / N)$. Choose $\delta \in \Sigma(f) \backslash T$ where $T$ is the set of $\delta$ in $\Sigma(f)$ such that $2 / \delta$ is an integer.

Since $u_{f}$ is nowhere vanishing, we have

$$
\int_{V^{\prime}} \frac{1}{|g(z, w)|^{2 \delta}} d V(w) \sim \int_{V^{\prime}} \frac{1}{\left|p_{g}^{2}\right|^{\delta}} d V(w)
$$

where the implied constant depends on $f$ and $\delta$, but not on $g$. 
If $\delta<2 / N$, then (2.2) implies that (2.5) is finite, so that $\delta<c_{0}(g)$.

If $\delta>2 / N$, choose $r, 1 \leq r \leq M$, such that $\delta \in(2 /(N-r+1), 2 /(N-r))$. Then applying Proposition 1 to the right hand side of (2.5), we obtain, for every $z \in U^{\prime}$,

$$
\int_{V^{\prime}} \frac{1}{|g(z, w)|^{2 \delta}} d V(w) \sim \frac{1}{F_{r+1, g}(z)^{(N-r+1) \delta-2} F_{r, g}(z)^{2-(N-r) \delta}}
$$

Thus we see that $\delta \in \Sigma(g)$ if and only if

$$
n(r+1, g)((N-r+1) \delta-2)+n(r, g)(2-(N-r) \delta)<2 .
$$

Since $\delta \in \Sigma(f),(2.6)$ holds when $g=f$. But $n(k, g) \leq n(k, f)$ for all $k$. Thus $\delta \in \Sigma(g)$.

3. Proof of Conjecture B when $n=2$. Let $\mathcal{C}=\mathcal{C}(2)$. It suffices to show that for every $r>0$, the set $\{c \in \mathcal{C}: c \geq r\}$ satisfies the ascending chain condition.

If $f$ is holomorphic in a neighborhood of the origin, and if $f(0)=0$, then $\Sigma(f) \subseteq$ $(0,4)$. Moreover, if $f$ is a Weierstrass polynomial of degree $M$, i.e., an expression of the form $w^{M}+a_{1}(z) w^{M-1}+\cdots+a_{M}(z)$, then $c_{0}(f)<2 / M$. Since $2 / M<r$ for $M$ sufficiently large, it suffices to prove that for each $M>0$,

$$
\mathcal{C}(M)=\left\{c_{0}(f): f \text { is a Weierstrass polynomial of degree } M\right\}
$$

satisfies the ascending chain condition. Thus we fix $M>0$ and let $N=2 M$. We have shown that $\mathcal{C}(M) \subseteq[1 / M, 2 / M]$. It therefore suffices to show that for every integer $r$ such that $1 \leq r \leq M$, the set $\mathcal{C}(M) \cap[2 /(N-r+1), 2 /(N-r)]$ satisfies the ascending chain condition (since $[2 / N, 4 / N]$ is a union of such intervals).

Thus we fix $r$ such that $1 \leq r \leq M$, and we let $\delta \in(2 /(N-r+1), 2 /(N-r))$. Let $f$ be a Weierstrass polynomial of degree $M$. It follows from (2.6) that if $c$ is a positive real number, then $c \in \mathcal{C} \cap(2 /(N-r+1), 2 /(N-r))$ if and only if

$$
\begin{gathered}
n(r+1)((N-r+1) c-2)+n(r)(2-(N-r) c)=2 \\
\text { and } c \in\left(\frac{2}{(N-r+1)}, \frac{2}{(N-r)}\right)
\end{gathered}
$$

where we have denoted $n(r, f)$ by $n(r)$ for simplicity. Now (3.2) is equivalent to, via simple algebraic manipulations,

$$
\begin{gathered}
c=2 \frac{n(r+1)-n(r)+1}{n(r+1)(N-r+1)-n(r)(N-r)} \text { and } \\
n(r+1)>N-r \text { and } n(r)<N-r+1
\end{gathered}
$$

The fact that $n(r)<N-r+1$, together with (2.4) tells us that there are only finitely many possibilities for $n(r)$. For each such choice of $n(r)$, there may be infinitely many possibilities for the value of $n(r+1)$, but these possibilities must also satisfy (2.4). In particular, the possible values of $n(r+1)$ form a subset of

$$
X=\{n \in \mathbf{Q}: n(N !) ! \in \mathbf{Z}, n>N-r\} .
$$

Now fix $n(r)$ and let $n(r+1)$ range over $X$ in equation (3.3). The key observation is that as $m \in X$ tend towards infinity, the values of $c$ form a decreasing sequence, converging to $2 /(N-r+1)$. This proves Conjecture $B$, in the case $n=2$. It also shows that the limit points of $\mathcal{C}(2)$ are either 0 , or rational numbers of the form $2 / a$ 
with $a$ a positive integer. In other words, the limit set of $\mathcal{C}(2)$ is contained in $\mathcal{C}(1)$. In fact, it is equal to $\mathcal{C}(1)$, as we shall see in the next section*.

4. The limit set of $\mathcal{C}(n)$. It follows immediately from the definitions that $\mathcal{C}(n) \subseteq \mathcal{C}(n+1)$ for $n \geq 1$.

Proposition 2. If $c \in \mathcal{C}(n)$ then $c=\lim x_{m}$, where $x_{1}, x_{2}, \ldots \in \mathcal{C}(n+1)$ forms a strictly decreasing sequence.

Proof. Let $f$ be holomorphic in a neighborhood of the origin of $\mathbf{C}^{n}$ such that $c_{0}(f)=c$. Let $m$ be a sufficiently large positive integer and let $g_{m}\left(z_{1}, \ldots, z_{n}, w\right)=$ $w^{m}-f$. Then

$$
\int_{B} \frac{1}{\left|w^{m}-f\right|^{2 \delta}} d V(w) \sim \frac{1}{|f|^{2(\delta-1 / m)}}
$$

Thus $c_{0}\left(g_{m}\right)=c+\frac{1}{m}$.

REMARKS. 1. The same proofs apply in dimension two to give the analogues of Conjectures $A$ and $B$ in the real-analytic setting, using the results in $[P S S]$. In dimensions 3 and higher, it is known that even the weaker conjecture $A_{\epsilon}$ is not true, due to a counterexample of Varchenko.

2. It is tempting to speculate that $\mathcal{C}(n)$ is exactly the limit set of $\mathcal{C}(n+1)$. This was also suggested earlier by Kollár, who also formulated Proposition 2 in [K2].

3. More specifically, it is likely that the structure of $\mathcal{C}(n+1)$ is determined inductively by that of $\mathcal{C}(n)$ in the manner suggested above:

$$
\mathcal{C}(n+1)=\mathcal{C}(n) \cup \bigcup_{c \in \mathcal{C}(n), 1 \leq m<\infty} x(c, m)
$$

where for each $c$, the $x(c, m)$ forms a strictly decreasing sequence such that

$$
\lim _{m \rightarrow \infty} x(c, m)=c .
$$

ACKnowledgements. The authors would like to thank Professor J. Kollár for informing them about the works of Shokurov, Alexeev, and Igusa, and for providing them with the references $[\mathrm{S}],[\mathrm{A}],[\mathrm{I}]$, and [K1]. They would also like to thank Professor S.T. Yau for his encouragement and for providing them with references on KählerEinstein metrics.

\section{REFERENCES}

[A] V. A. Alexeev, Two-dimensional terminations, Duke Math. J., 69 (1993), pp. 527-545.

[CY] S. Y. CHENG AND S. T. YAU, Inequality between Chern numbers of singular Kähler surfaces and characterization of orbit space of discrte group of $S U(2,1)$, Contemporary Math., 49 (1986), pp. 31-44.

[DK] J. P. Demailly AND J. Kollár, Semi-continuity of complex singularity exponents and Kähler-Einstein metrics on Fano orbifolds, arXiv:math.AG/9910118.

*In dimension $n=2$, the exponents $c_{0}(f)$ were determined by Igusa [I] to be of the form $c_{0}(f)=\frac{1}{m}+\frac{1}{n}, m, n \in \mathbf{N}^{*}$, for irreducible power series $f \in \mathbf{C}[[x, y]]$. But for a general holomorphic function $f$ in two variables, they can be more complicated, as we just saw. For example, for $f(x, y)=$ $y\left(y^{2}+x^{89}\right)$, the exponent $c_{0}(f)$ is given by $c_{0}(f)=\frac{91}{267}$, which cannot be expressed as $\frac{1}{m}+\frac{1}{n}$ for any integers $m, n$. 
[I] J. I. IGUSA, On the first terms of certain asymptotic expansions, in Complex and Algebraic Geometry, Iwanami Shoten, 1977, pp. 357-368.

[K1] J. Kollár (with 14 coauthors), Flips and Abundance for Algebraic Threefolds, Astérisque $211,1992$.

[K2] J. Kollár, Singularities of pairs, in Proceedings of Symposia in Pure Mathematics 62, American Mathematical Society, 1997, pp. 221-285.

[PS] D. H. Phong AND J. STURM, Algebraic estimates, stability of local zeta functions, and uniform estimates for distribution functions, Preprint, January 1999, to appear in Ann. of Math..

[PSS] D. H. PhONG, E. M. STEIn, AND J. STuRM, On the growth and stability of real-analytic functions, Amer. J. Math., 121 (1999), pp. 519-554.

[Sh] V. Sнокurov, 3-fold log flips, Isz. Russ. A. N. Ser. Mat., 56 (1992), pp. 105-203.

[Si] Y. T. SIU, The existence of Kähler-Einstein metrics on manifolds with positive anticanonical bundle and a suitable finite symmetry group, Ann. of Math., 127 (1987), pp. 585-627.

[T1] G. TIAN, On Kähler-Einstein metrics on certain manifolds with $c_{1}(M)>0$, Inventiones Math., 89 (1987), pp. 225-246.

[T2] G. TIAN, On Calabi's conjecture for complex surfaces with positive first Chern class, Inventiones Math., 101 (1992), pp. 101-172.

[TY1] G. TIAN AND S. T. YAU, Existence of Kähler-Einstein metrics on complete Kähler manifolds and their applications to algebraic geometry, in Mathematical aspects of String Theory, Adv. Ser. Math. Phys. 1, World Scientic Publ., Singapore, 1986, pp. 574-628.

[TY2] G. TIAN AND S. T. YAU, Complete Kähler manifolds with zero Ricci curvature II, Inventiones Math., 106 (1991), pp. 27-60.

[Y1] S. T. YAU, Open problems in geometry, Proceedings of Symposia in Pure Mathematics, 54 (1993), pp. 1-28.

[Y2] S. T. YAU, Review of Kähler-Einstein metrics in algebraic geometry, Israel Mathematical Conference Proceedings, 9 (1996), pp. 433-443. 